\title{
Correction to: COVID-19 outbreak situation and its psychological impact among surgeons in training in France
}

\author{
Maher Abdessater ${ }^{1} \cdot$ Morgan Rouprêt $^{1} \cdot$ Vincent Misrai $^{2} \cdot$ Ugo Pinar $^{1} \cdot$ Xavier Matillon $^{3} \cdot$ Bastien Gondran-Tellier $^{4}$. \\ Lucas Freton $^{5} \cdot$ Maxime Vallée $^{6} \cdot$ Inès Dominique ${ }^{7} \cdot$ Margaux Felber $^{1} \cdot$ Zine-Eddine Khene $^{5} \cdot$ Edouard Fortier $^{8}$. \\ François Lannes ${ }^{4} \cdot$ Clément Michiels $^{9} \cdot$ Tristan Grevez $^{10} \cdot$ Nicolas Szabla $^{11} \cdot$ Florian Bardet $^{12} \cdot$ Kevin Kaulanjan $^{13}$. \\ Emilien Seizilles de Mazancourt ${ }^{3} \cdot$ Guillaume Ploussard $^{14} \cdot$ Benjamin Pradere $^{10,15}$
}

Published online: 23 May 2020

(c) Springer-Verlag GmbH Germany, part of Springer Nature 2020

Correction to: World Journal of Urology https://doi.org/10.1007/s00345-020-03207-x

In the original publication of the article, the first and last name of the first author were interchanged. The correct name of the author should be as given below:

First Name: Maher

Last Name: Abdessater

The original article has been updated accordingly.
The original article can be found online at https://doi.org/10.1007/ s00345-020-03207-x.

Morgan Rouprêt

morgan.roupret@aphp.fr

1 Sorbonne Université, GRC n5, Predictive Onco-Urology, Ap-Hp, Hôpital Pitié-Salpêtrière, Urology, 75013 Paris, France

2 Clinique Pasteur, 31300 Toulouse, France

3 Department of Urology and Transplantation, Edouard Herriot Hospital, Hospices Civils de Lyon, Lyon, France

4 Department of Urology, La Conception University Hospital, Assistance-Publique Marseille, Marseille, France

5 Department of Urology, University Hospital of Rennes, Rennes, France

6 Department of Urology, Poitiers University Hospital, Poitiers, France

7 Department of Urology, Groupe Hospitalier Diaconesses Croix Saint-Simon, Paris, France
8 Department of Urology, Montpellier University Hospital, Montpellier, France

9 Department of Urology, Bordeaux University Hospital, Bordeaux, France

10 Department of Urology, CHRU Tours, Francois Rabelais University, Tours, France

11 Department of Urology and Transplantation, Caen University Hospital, Caen, France

12 Department of Urology, Dijon University Hospital, Dijon, France

13 Department of Urology, CHU Pointe à Pitre, Guadeloupe, France

14 Department of Urology, Ramsay Santé, Clinique la Croix du Sud, Quint Fonsegrives, France

15 Department of Urology, Comprehensive Cancer Center, Medical University of Vienna, Vienna, Austria 